\title{
Off-Pump Coronary Artery Bypass Grafting Improves Early Clinical Outcomes Including Operative Mortality
}

\author{
Murali P Vettath, MBBS, DNB, MCh, ${ }^{1}$ Madhu Ravisankar, MBBS, DNB, ${ }^{2}$ Tomislov Kopjar, MD, ${ }^{3}$ \\ AV Kannan, MD, ${ }^{2}$ Nitin Gangadharan, MD $^{2}$ \\ ${ }^{1}$ Chairman of Cardiovascular and Thoracic Centre and ${ }^{2}$ Department of Cardiovascular and Thoracic Surgery, MEITRA \\ Hospital Kozhikode, Kerala India; ${ }^{3}$ Department of Cardiac Surgery, University of Zagreb School of Medicine, University \\ Hospital Centre Zagreb, Zagreb, Croatia
}

\section{ABSTRACT}

Background: Whether coronary artery bypass grafting $(\mathrm{CABG})$ should be performed on- or off-pump remains a matter of debate. We aim to present our experience with offpump CABG. Early clinical outcome and adverse events were analyzed over the time course of the study.

Methods: A total of 4310 patients undergoing isolated off- pump CABG from January 2002 until December 2016 at the Malabar Institute of Medical Sciences in India were included. Preoperative, intraoperative, and postoperative, as well as follow-up data were prospectively collected. To analyze the differences of patient characteristics and outcomes over time, five-year periods were created (early: 2002-2006; middle: 2007-2011; late: 2012-2016). Traditional techniques of quality control monitoring were applied.

Results: The mean age of our patients was $59 \pm 9$ years, and $13 \%$ (533) were female. Postoperative mortality was observed in $0.7 \%$ (25), acute renal failure and stroke in $0.2 \%$ (8) each, and mediastinitis in $1.2 \%$ (53) of the patients. Despite the progressive worsening of the patient risk profile, significant improvement in mortality was observed over time, while stroke, acute renal failure, and mediastinitis remained similar. Continuous quality control monitoring revealed that the system was within the control boundaries for the entire period of the study. The current probability of 30-day mortality or conversion to on-pump CABG is about $0.5 \%$.

Conclusion: Off-pump CABG is safe and effective for patients undergoing $\mathrm{CABG}$. It can provide superior results compared to on-pump CABG, particularly when performed by a dedicated off-pump surgeon.

\section{INTRODUCTION}

There is much controversy surrounding the methods of performing coronary artery bypass grafting (CABG). The question of whether CABG should be performed on- or

Received March 20, 2018; received in revised form April 26, 2018; accepted April 27, 2018.

Correspondence: Murali P Vettath, MBBS, DNB, MCh, Meitra Hospital, Karaparamba - Kunduparamba bypass road, Kozhikode, Kerala, 673005, India;0091-9846247089 (e-mail: murali.vettath@gmail.com). off-pump remains unanswered [Deppe 2016]. Several comparative studies and multicenter trials have surfaced. Benefits of off-pump CABG in terms of death, myocardial infarction $(\mathrm{MI})$, and stroke are related to patient risk profile, suggesting that off-pump CABG should strongly be considered in highrisk patients [Kowalewski 2016]. However, in low risk groups, there has been no superiority shown for either technique at long term follow-up [Lamy 2016].

In 2002, our unit adopted off-pump CABG as the procedure of choice for coronary revascularization. As it is a single surgeon center, there are more technical constants than in a teaching hospital, where more surgeons operate. Previously, we published details regarding our off-pump technique and how we avoid conversions, and the role of the intra-aortic balloon pump (IABP) [Vettath 2016; Vettath 2013]. Although the safety and the efficacy in off-pump CABG have been reported to be comparable to on-pump CABG, off-pump CABG is a more of a skill based procedure. Hence, the results in different centers have not been unanimous.

We have not been performing on-pump CABG for the past ten years, and therefore we could not benchmark against it. Over the years, we have worked to improve the outcomes of our off-pump CABG patients, and to maintain a low level of adverse events. In this article, we would like to present our experience with off-pump CABG. Early clinical outcomes and adverse events were analyzed over the time course of the study, and traditional techniques of quality control monitoring were applied.

\section{PATIENTS AND METHODS}

\section{Study Population}

From January 2002 until December 2016, patients were referred to the senior author (Murali Prabhakaran Vettath) for surgical treatment of coronary artery disease at the Malabar Institute of Medical Sciences (MIMS) in India. Over the 15-year period, 4310 patients underwent isolated off-pump CABG. Preoperative, intraoperative, and postoperative outcomes and follow-up data were collected prospectively, and retrospectively analyzed. To analyze the differences of patient characteristics and outcomes over time, five-year periods were created. Patients that were operated on from 2002 until 2006 were in the early period, 20072011 in the middle period, and 2012-2016 in the late period. 
Table 1. Baseline Demographics and Patient Characteristics Across Periods

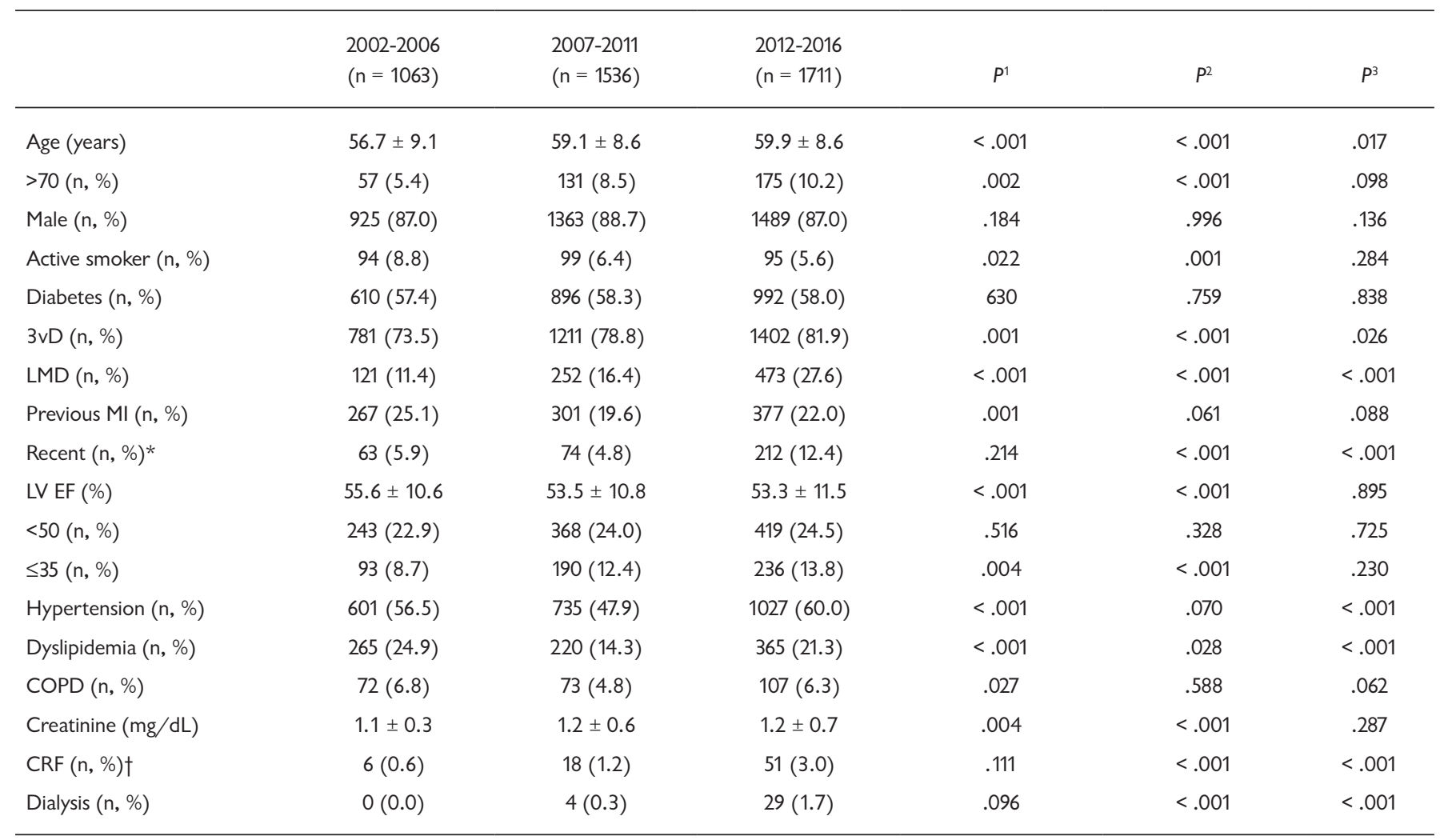

3vD indicates three vessel disease; COPD, chronic obstructive pulmonary disease; CRF, chronic renal failure; EF, ejection fraction; LMD, left main disease; LV, left ventricle; $\mathrm{Ml}$, myocardial infarction.

*Myocardial infarction occurring within two weeks of surgery; $\nmid$ Preoperative serum creatinine $\geq 2.3 \mathrm{mg} / \mathrm{dL}$ or dialysis.

$P^{1}$-value: early versus middle cohort. $P^{2}$-value: early versus late cohort. $P^{3}$-value: middle versus late cohort.

The procedures followed were in accordance with the ethical standards of the Helsinki Declaration (1964, amended most recently in 2008) of the World Medical Association. The study design conforms to the ethical standards currently applied in India.

The patient's written consent form was not obtained due to the retrospective nature of the study. The indication for surgery was significant flow-limiting coronary artery stenosis involving one or more of the major coronary arteries that was not suitable for percutaneous coronary intervention, accompanied either by angina refractory to medical therapy, targeted myocardial viability, inducible ischemia, or history of life-threatening arrhythmia. A single-surgeon practice was used to prevent variability in surgical technique from causing bias in data interpretation, and to avoid problems with missing data. The intention to treat for the entire cohort was off-pump CABG. Some patients were converted to on-pump either during anesthesia induction, assessment of the heart, or coronary grafting. Patients converted to on-pump CABG were not excluded from the analysis. Combined procedures involving other cardiac pathologies, such as combined valve replacements with $\mathrm{CABG}$, or other surgical pathologies which required interventions, were not included

\section{Off-Pump Coronary Artery Bypass Grafting}

Standardized protocol for off-pump CABG developed at MIMS was followed. It included a discontinuation of all blood pressure-lowering medication on the morning of surgery (including beta blockers). Every patient had a femoral arterial line and a central venous line introduced prior to the commencement of the procedure under general anesthesia. Bearing in mind that inotropes will only make an ischemic heart more ischemic, we substituted intraoperative use of inotropes with atropine, to increase heart rate and maintain hemodynamic stability. If atropine proved to be insufficient, and in case of hemodynamic instability at any time during the procedure, the femoral line was used for a sheath-less insertion of an IABP prior to initiation of inotropes.

If malignant ventricular arrhythmias occurred, they were treated by direct current shock, an intravenous lidocaine bolus, and the introduction of an amiodarone infusion. Plasma electrolytes and $\mathrm{pH}$ balance were maintained in the normal range. During distal grafting, temporary lowering of head, volume resuscitation, and intravenous administration of atropine were used to achieve target mean arterial blood pressure $>80 \mathrm{mmHg}$. The IABP was used only if the patient developed hemodynamic compromise, and was used only prior to initiation of any inotropes. In our earlier 




Figure 1. Cumulative sum (CUSUM) chart of 30-day mortality or conversion to on-pump coronary artery bypass grafting with alarm, alert, and reassurance boundary lines, and three predefined study periods (early: 2002-2006; middle: 2007-2011; late: 2012-2016).

experience, once IABP was placed, it was kept in for 24 - 48 hours. Since 2008, all the patients who had IABP inserted had it removed prior to exiting the operating theatre.

After pericardiotomy, cardiac manipulation was avoided and only the left anterior descending artery (LAD) was inspected and palpated. Patients were fully heparinized and converted with protamine sulfate after grafting was completed. Warm saline-soaked gauze pads were used to position the heart. Occasionally, a deep pericardial stay suture was used to aid in the positioning of the heart. Various types of stabilizers, blowers, and mist blowers were used over the years to aid in the stabilization of the heart. We have also developed our own reusable stabilizer: the Simple Indian Made Stabilizer (SIMS). Video 1 (https://vimeo.com/268972240) shows the SIMS being used for LIMA to LAD anastomosis, and video 2 (https://vimeo.com/268972342) shows the anastomosis of a vein graft to an intramuscular obtuse marginal branch of circumflex using SIMS. The SIMS can be used as a suction stabilizer or as a mechanical stabilizer without suction.

Complete revascularization was attempted in all patients. First, the LAD grafting was attempted, preferably with the left internal mammary artery (LIMA). After grafting the LAD, the heart was lifted and the remaining target vessels were assessed and grafted either with venous or arterial conduits. Then the top end anastomoses were performed. Occasionally, in case of a severely calcified aorta the Vettath's Anastomotic Obturator (VAO) was used to aid in the performance of the proximal anastomosis, without using the side clamp (Figure 3 shows the top end anastomosis in progress; video 3 (https://vimeo.com/268972415) shows two top end anastomosis of vein graft being performed using VAO). After the procedure, the patients were taken to the cardiac intensive care unit.

\section{Postoperative Outcome Definitions}

The following outcomes were looked at in the early postoperative period. Operative mortality was defined as death



Figure 2. Probability of 30-day death or conversion to on-pump coronary artery bypass grafting by the chronological sequence number of patients for the entire patient cohort with three predefined study periods (early: 2002-2006; middle: 2007-2011; late: 2012- 2016).

during the indexed hospitalization, regardless of the length of stay, or any death within 30 days of surgery. Mediastinitis was considered as any culture-positive infection involving the muscle, sternum, or mediastinum leading to instability, exploration, and debridement of the wound, and requiring antibiotics for treatment. Stroke was defined as a new, central neurological deficit persisting for more than 24 hours. Acute renal failure was defined as a new need for hemodialysis. There were no patients lost to follow-up, and the final dead or alive status was determined for all patients.

\section{Statistical Analysis}

The continuous data are presented as mean values \pm standard deviation (SD) for normally distributed variables, or medians with interquartile range (IQR) otherwise. Categorical variables are presented as frequency and proportions, and compared with the Pearson $\chi^{2}$ or Fisher's exact test when frequency is less than 10. Differences between means were tested using the Independent-Samples T Test or Mann-Whitney U test, where appropriate. When comparing continuous data across the three

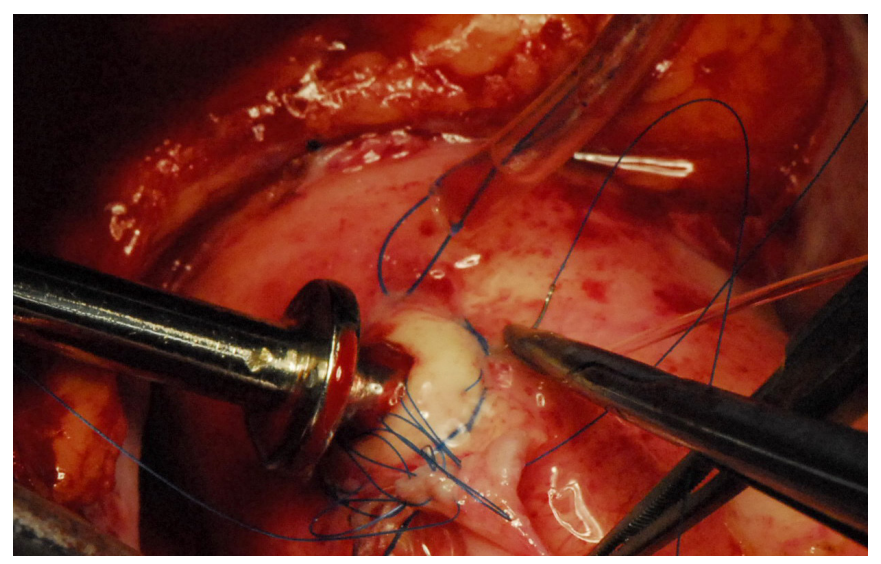

Figure 3. The top end anastomosis in progress. 
Table 2. Operative Data Across Periods

\begin{tabular}{|c|c|c|c|c|c|c|}
\hline Arterial & $0.76 \pm 0.43$ & $0.82 \pm 0.40$ & $0.84 \pm 0.38$ & .002 & $<.001$ & .154 \\
\hline Venous & $2.2 \pm 1.2$ & $2.2 \pm 1.0$ & $2.1 \pm 0.8$ & .975 & .252 & .283 \\
\hline Conversion (n, \%) & $18(1.7)$ & $1(0.1)$ & $0(0)$ & $<.001$ & $<.001$ & .473 \\
\hline
\end{tabular}

IABP indicates intra-aortic balloon pump; LIMA, left internal mammary artery.

$P^{1}$-value: early versus middle cohort. $P^{2}$-value: early versus late cohort. $P^{3}$-value: middle versus late cohort.

time periods, analysis of variance using the One-Way ANOVA procedure was performed. To determine which means differed, a post hoc Tukey's honestly significant difference test was used.

Binary logistic regression analysis was used to explore how the rate of procedural failure decreased over the chronological patient sequence number, and to provide current procedural failure estimates. The definition of procedural failure for each operation was 30-day mortality or conversion to onpump CABG. The results of binary logistic regression analysis were presented as odds ratio (OR) with $95 \%$ confidence intervals (CI). Graphical analysis was then performed by plotting the probability of procedural failure versus the chronological sequence of each case (experience). The $P$-value was set at less than .05 to detect statistical significance. Statistical analysis was carried out using the statistical software IBM SPSS Statistics (IBM Corp: Armonk, NY).

A cumulative sum (CUSUM) quality control chart of sequential monitoring was created to evaluate the procedural success/failure rate over time associated with off-pump CABG. The definition of procedural failure was the same as the one used in the binary logistic regression analysis (30-day mortality or conversion to on-pump CABG). The statistical principles were adapted from the comprehensive tutorial by Rogers et al [Rogers 2004]. To describe it briefly, CUSUM is a graph of the cumulative number of failures over experience. The cumulative number of failures either remains unchanged in case of procedural success, or increases if a failure occurs.

Immediate visual interpretation of the graph is aided with control boundary (alarm, alert and reassurance) lines. Upper control lines are constructed to detect an increase in failures to the unacceptable rate. If the graph intersects the lower boundary (reassurance) line it can be concluded that the failure rate is less than or equal to the acceptable, and the performance is considered proficient. When a graph remains between these boundaries, the evidence remains inconclusive, and monitoring should continue. The probability of falsepositive, type I error $(\alpha)$ and false-negative, type II error $(\beta)$ were set at $1 \%$ for the alarm and $5 \%$ for the alert and reassurance lines. Acceptable failure rate was set at $2.5 \%$, while unacceptable was $5 \%$.
RESULTS

\section{Entire Cobort}

The mean age of the 4310 patient cohort was $59 \pm 9$ years, and $13 \%(533)$ were female patients. Most of the patients, $79 \%$ (3394) had triple vessel disease. The mean ejection fraction of the left ventricle for the entire cohort was $54 \pm$ $11 \%$, and $22 \%$ (945) had previous myocardial infarction. Common comorbid conditions were diabetes in 58\% (2498) of the patients, and hypertension in 55\% (2363). On average, a patient received $3.0 \pm 0.9$ grafts: $0.8 \pm 0.4$ arterial and 2.1 \pm 1.0 venous. The left internal mammary artery was used in $81 \%$ (3474) of cases. In $4.8 \%$ (207) of the patients, IABP was used to maintain hemodynamic stability. Some patients $(0.4 \%$ [19]) were converted to on-pump CABG. The 30-day mortality of the entire cohort was $0.6 \%$ (25). Eight patients $(0.2 \%)$ developed postoperative stroke. Mediastinitis occurred in $1.2 \%(53)$ of the patients, and acute renal failure in $0.2 \%(8)$.

\section{Outcomes Across Periods}

Preoperative demographics and patient characteristics across the three time periods are described in Table 1 . A significant increase from the early (2002-2006) to the late (20122016) period can be observed for age, severity of coronary pathology (triple vessel disease and left main disease), recent myocardial infarction, renal disease (creatinine, chronic renal failure, and dialysis), and left ventricular impairment, whereas a decrease of the number of active smokers and dyslipidemia was observed. Intraoperative data are provided in Table 2. The average number of grafts per patient did not differ significantly over time. An increase in the number of arterial grafts, left internal mammary artery use, and IABP use was observed in the late period when compared to early, whereas conversions decreased. Postoperative outcome data are provided in Table 3. Significant differences between time periods were observed for mortality, but stroke, acute renal failure, and mediastinitis were similar.

\section{Analysis of Procedural Failure Over Time}

As mentioned previously, the overall 30-day mortality rate was $0.6 \%(25)$, and the conversion rate was $0.4 \%(19)$. 
Table 3. Postoperative Outcome Comparison Across Periods

\begin{tabular}{lccccc}
\hline & $\begin{array}{c}2002-2006 \\
(\mathrm{n}=1063)\end{array}$ & $\begin{array}{c}2007-2011 \\
(\mathrm{n}=1536)\end{array}$ & $\begin{array}{c}2012-2016 \\
(\mathrm{n}=1711)\end{array}$ & $P^{1}$ & $P^{3}$ \\
\hline Stroke (n, \%) & $1(0.1)$ & $1(0.1)$ & $6(0.4)$ & 1.000 & .262 \\
ARF (n, \%) & $1(0.1 \%)$ & $3(0.2)$ & $4(0.2)$ & .649 & .655 \\
Mediastinitis (n, \%) & $12(1.1 \%)$ & $15(1.0)$ & $26(1.5)$ & .707 & .389 \\
Mortality (n, \%) & $10(0.9)$ & $2(0.1)$ & $13(0.8)$ & .005 & .600 \\
\hline
\end{tabular}

ARF indicates acute renal failure.

$P^{1}$-value: early versus middle cohort; $P^{2}$-value: early versus late cohort; $P^{3}$-value: middle vs late cohort.

This was used to calculate the procedural failure rate of $1 \%$ (43), which was defined as 30-day mortality or conversion to on-pump CABG. The CUSUM chart for the entire cohort of 4310 patients is presented in Figure 1. The chart shows that the cumulative failures remain well below the alarm line, which set out to detect an increase in procedural failure from the acceptable 2.5 to the unacceptable $5 \%$ with $99 \%$ confidence. Overall, the failure rate is less than or equal to $2.5 \%$, and the performance is considered proficient. In the early period, around the experience of 200 cases, the CUSUM line touches the upper boundary Alert line, indicating a possible increase in failure rate to $5 \%$ with $95 \%$ confidence.

Increasing patient sequence led to a significant decrease in odds of procedural failure $(\mathrm{OR}=1.000,95 \%$ CI 0.999 to $1.000 ; P<.001)$. For each case, the odds of procedural failure decreased by $0.05 \%$. Figure 2 displays a progressively decreasing probability of procedural failure over chronological patient sequence. Significant differences in the mean probabilities of failures were observed between periods for all comparisons. The extent of decline in probability was $1,0.7$, and $0.4 \%$ for the early, middle, and late periods, respectively.

\section{DISCUSSION}

Our study presents a large series of off-pump CABG patients undergoing operation at a single center in India. The entire 4310 patient cohort was referred to a single surgeon dedicated to off-pump CABG. Excellent early clinical endpoints, including operative mortality, stroke, acute renal failure, and mediastinitis were observed over the time course of the study. We identified a clear improvement in outcome over time, despite the progressive worsening of the risk profile of the patients.

The evolution of less invasive techniques for heart surgery led to the resurfacing of off-pump CABG around the turn of the century, with aims to decrease perioperative complications that could possibly be related to the use of CPB and to cross-clamping of the aorta. In 2002, we started performing off-pump CABG at MIMS. Ever since then, it has been adopted as the procedure of choice for patients referred to us with coronary artery disease. Initially, we restricted this procedure to patients requiring fewer grafts. We also limited the procedure to those with certain comorbidities such as renal failure, calcified aorta, cerebrovascular disease, and other cases where we thought that the use of CPB might be contraindicated. Presently, we have operated on $\approx 4500$ coronary patients without the use of $\mathrm{CPB}$. The most recent conversion to on-pump CABG was in 2007. What we find to be crucial in the prevention of going on pump is the appropriate usage of IABP, as we described previously [Vettath 2013].

In 2009, off-pump procedures accounted for $21 \%$ of CABG in the United States, according to the data available from the Society of Thoracic Surgeons adult cardiac surgery database [ElBardissi 2012]. These rates are similar to the ones reported in 2011, in the 4th European Association for Cardio-Thoracic Surgery adult cardiac surgery database report [Bridgewater 2011]. Unlike in Europe and the United States, the rate of off-pump CABG is known to be relatively high in India [Saha 2014]. The importance of beating heart surgery and the associated cost reduction of CABG is even greater in India than what it is in Europe or the United States, considering the country's limited resources and poor health insurance coverage [Saha 2014].

Off-pump CABG surgery has always been a skill-based procedure with an extensive learning curve. Hence, the results have not been consistent across centers or within a center at various points in time. Despite these limitations, our results have improved over the years. Previously, after auditing our results, we noticed that our learning curve for offpump CABG extended beyond the first 500 patients [Vettath 2016]. This can easily be confirmed when looking at Figure 2. The greatest drop in probability of procedural failure was observed during the early period of the study, which extends further than the initial 500 cases. The probability of 30-day mortality or conversion to on-pump for the most recent period of our study is $0.5 \%$.

The unique technical challenges associated with off-pump CABG have raised concerns about a possible worse outcome during the adoption process of the technique [Song 2003]. The learning curve associated with the adoption of a new surgical technique shows the steepest slope during the initial stages of the adoption process. Absence of our own onpump CABG patients prevented us from having a benchmark against it. Therefore, a technique of sequential quality control monitoring helped us visualize the success/failure rate over time. Compared to other studies, the bar to detect a 
system out of control was set much higher in our study [Murzi 2012; Novick 2002]. Albeit, the failure rate observed in our study was below the upper boundary line for the entire period of monitoring. Following the experience of about 600 cases, the CUSUM line intersected with the reassurance boundary line and remained well below it for the remaining duration of monitoring.

Generally, the operative (30-day) mortality of on-pump CABG is about $2 \%$. An additional 5 to $7 \%$ of patients are considered to have myocardial infarction, stroke, and renal failure requiring dialysis. Early initiation of IABP lead to an improvement in clinical outcome of our patients, with a significant reduction in conversion rate, perioperative $\mathrm{MI}$ and renal failure [Vettath 2016]. The standardization of the approach to a patient with ischemic heart disease and how we do off-pump CABG has consistently brought down the operative (30-day) mortality rate of our patients, lowering it to less than $1 \%$, which is superior to 30 -day results published in large randomized controlled trials to date [Diegeler 2013; Houlind 2012; Shroyer 2009].

Recently, Varma et al published their 11-year experience with 4024 primary isolated on-pump CABG from a neighboring center in India, the Sree Chitra Tirunal Institute for Medical Sciences and Technology (SCTIMST) [Varma 2014]. The SCTIMST is a teaching hospital where several surgeons operate. In their study, they report on early outcomes of patients from the same region and period, and with a similar profile as the ones in our off-pump cohort. Their reported 30-day mortality and the need for new onset hemodialysis was higher (2.2 and $0.5 \%$, respectively). Stroke rate was similar, and mediastinitis $(0.7 \%)$ was lower.

Several observational studies comparing off-pump to onpump CABG have already been published. More recently, randomized controlled trials have also emerged. One would expect that the debate of superiority has been concluded, however, that is far from true. Lower rates of long-term survival associated with off-pump CABG have been reported in some nonrandomized trials [Kim 2014]. Studies reporting on lower rates of long-term survival together with those reporting on higher rates of repeat revascularization were the basis of the suggestion of some authors that off-pump CABG be abandoned [Lazar 2013; Hannan 2007].

As for the evidence available from randomized trials, the largest randomized trial to date is the CABG Off or On Pump Revascularization Study (CORONARY), involving 4752 patients recently reported on five-year follow-up [Lamy 2016]. Although the early 30-day outcome in the CORONARY study showed fewer grafts in the off-pump group and higher rate of incomplete revascularization, at five years there was no significant difference in any outcome, and the authors concluded that both procedures are equally effective and safe [Lamy 2016; Lamy 2012]. The results were consistent even among patients who crossed over to the other procedure or received an incomplete revascularization during their CABG procedure [Lamy 2016]. Other large randomized controlled trials comparing off-pump and on-pump CABG were published, though they have not reported on long-term outcomes [Diegeler 2013; Houlind 2012; Shroyer 2009].
Recently, two articles were published comparing survival of patients undergoing on-pump and off-pump CABG. Smart et al state in their study that, "Statistically, on-pump CABG appeared to offer superior long-term survival, although the clinical significance of this may be more uncertain" [Smart 2018]. Again, Shroyer et al showed that "Off-pump CABG led to lower rates of 5-year survival and event-free survival than on-pump CABG" [Shroyer 2017].

In spite of so many publications not supporting off-pump CABG surgery, we have been able to show that in the proper hands, off-pump CABG not only delivers lower mortality, but also reasonable long term survival.

Our study has several limitations. It is a retrospective analysis of observationally collected data, and thus, there might have been selection bias in choosing off-pump in performing CABG. To address this, we included all the patients undergoing off-pump CABG during a predefined period. The study reflects the practice of a single surgeon over a period of 15 years, and the evolution in the complexity of patients. Teaching institutions and centers where multiple surgeons operate might face more difficulty in achieving the same results. Over the time of the study, early postoperative management of patients in the intensive care unit must have evolved, contributing to the decrease in mortality. Our study results do support the use of off-pump CABG for the surgical treatment of coronary artery disease, but a direct comparison between off- and on-pump CABG was not feasible.

\section{CONCLUSION}

In conclusion, the results of our study report excellent short term outcomes of off-pump CABG. Low rates of adverse events were maintained throughout all three study periods, despite progressive worsening of the patient profile over time. Considering our hospital setup, the importance of a dedicated off-pump surgeon is emphasized. We advocate for the off-pump approach in patients undergoing surgery for coronary artery disease, particularly in situations when it is done by a dedicated off-pump surgeon.

\section{ACKNOWLEDGEMENTS}

We acknowledge the help of Ms. Nimmi Nanoo, Mr. Arun, Mr. Bipin, and Mr. Jayaprakash in compiling and assisting in the data entry over the years.

\section{REFERENCES}

Bridgewater B, Kinsman R, Walton P, et al. 2011. The 4th European Association for Cardio-Thoracic Surgery adult cardiac surgery database report. Interact Cardiovasc Thorac Surg 12:4-5.

Deppe AC, Arbash W, Kuhn EW, et al. 2016. Current evidence of coronary artery bypass grafting off-pump versus on-pump: A systematic review with meta-analysis of over 16900 patients investigated in randomized controlled trials. Eur J Cardio-Thoracic Surg 49:1031-41.

Diegeler A, Börgermann J, Kappert U, et al. 2013. Off-pump versus onpump coronary-artery bypass grafting in elderly patients. N Engl J Med 368:1189-98. 
ElBardissi AW, Aranki SF, Sheng S, et al. 2012. Trends in isolated coronary artery bypass grafting: an analysis of the Society of Thoracic Surgeons adult cardiac surgery database. J Thorac Cardiovasc Surg $143: 273-81$.

Hannan EL, Wu C, Smith CR, et al. 2007. Off- pump versus on-pump coronary artery bypass graft surgery: Differences in short-term outcomes and in long-term mortality and need for subsequent revascularization. Circulation 116:1145-52.

Houlind K, Kjeldsen BJ, Madsen SN, et al. 2012. On-pump versus off-pump coronary artery bypass surgery in elderly patients: Results from the danish on-pump versus off-pump randomization study. Circulation 125:2431-9.

Kim JB, Yun S-C, Lim JW, et al. 2014. Long-term survival following coronary artery bypass grafting: off-pump versus on-pump strategies. J Am Coll Cardiol 63:2280-8.

Kowalewski M, Pawliszak W, Malvindi PG, et al. 2016. Off-pump coronary artery bypass grafting improves short-term outcomes in high- risk patients compared with on-pump coronary artery bypass grafting: Metaanalysis. J Thorac Cardiovasc Surg 151:60-77.e58.

Lamy A, Devereaux PJ, Prabhakaran D, et al. 2016. Five-year outcomes after off-pump or on-pump coronary-artery bypass Grafting. N Engl J Med 375:2359-68.

Lamy A, Devereaux PJ, Prabhakaran D, et al. 2012. Off- pump or on-pump coronary-artery bypass grafting at 30 days. N Engl J Med 366:1489-97.

Lazar HL. 2013. Should off-pump coronary artery bypass grafting be abandoned? Circulation 128:406 LP-413.

Murzi M, Cerillo AG, Bevilacqua S, et al. 2012. Enhancing departmental quality control in minimally invasive mitral valve surgery: A single-institution experience. Eur J Cardio-Thoracic Surg 42:500-6.

Novick RJ, Fox SA, Stitt LW, et al. 2002. Assessing the learning curve in off-pump coronary artery surgery via CUSUM failure analysis. Ann Thorac Surg 73:S358-62.

Rogers CA, Reeves BC, Caputo M, et al. 2004. Control chart methods for monitoring cardiac surgical performance and their interpretation. J Thorac Cardiovasc Surg 128:811-9.

Saha KK. 2014. Off-pump coronary artery bypass grafting in India. Indian Heart J 66:203-7.

Shroyer AL, Grover FL, Hattler B, et al. 2009. On-pump versus offpump coronary-artery bypass surgery. N Engl J Med 361:1827-37.

Shroyer AL, Hattler B, Wagner TH, et al. 2017. Five-year outcomes after on-pump and off-pump CABG. N Engl J Med 377:623-32.

Smart NA, Dieberg G, King N. 2018. Long-term outcomes of on- versus off-pump coronary artery bypass grafting. J Am Coll Cardiol. 71: 983-91.

Song HK, Petersen RJ, Sharoni E, et al. 2003. Safe evolution towards routine off-pump coronary artery bypass: Negotiating the learning curve. Eur J Cardio- Thoracic Surg 24:947-52.

Varma PK, Kundan S, Ananthanarayanan C, et al. 2014. Demographic profile, clinical characteristics and outcomes of patients undergoing coronary artery bypass grafting-retrospective analysis of 4,024 patients. Indian J Thorac Cardiovasc Surg 30:272-7.

Vettath MP, Thazkuni IE, Vellachamy KA. 2013. Re-engineering in OPCAB - A Vettath's perspective. World J Cardiovasc Dis 3:33-44.

Vettath MP, Vellachamy KA, Gangadharan N. 2016. Role of intra-aortic balloon pump in off-pump coronary artery bypass - A Vettath Modification. World J Cardiovasc Dis 6:188-95. 